\begin{tabular}{|l|l|} 
Inif & ONAFT \\
Open Access
\end{tabular}

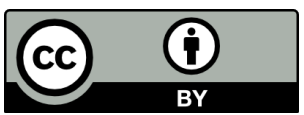

\title{
Квадратуры и аффинная геометрия на прямой
}

\author{
Н. Г. Коновенко \\ http://orcid.org/0000-0002-8631-0688
}

Аннотация $\mathrm{B}$ этой статье мы используем аффинную геометрию на прямой для построения обыкновенных дифференциальных уравнений, интегрируемых в квадратурах. Эти уравнения являются дифференциальными уравнениями для аффинных геометрических величин, допускающих аффинную группу симметрий. По теореме Ли-Бьянки эти уравнения интегрируются в квадратурах Приведены примеры, иллюстрирующие данный метод.

Ключевые слова Аффинная геометрия, дифференциальные инварианты, аффинные геометрические величины.

УДК 514.132

\section{1 Введение.}

Применение аффинной геометрии прямой к дифференциальным уравнениям [3] основывается не следующих наблюдениях:

1) Если обыкновенное дифференциальное уравнение второго порядка допускает в качестве симметрий 2-х мерную разрешимую алгебру $\mathfrak{a}$, являющуюся поднятием некоторого действия на прямой, то это означает, что на данной прямой задана аффинная структура, а решения данного уравнения являются аффинными геометрическими величинами. 
2) Если дифференциальный оператор, задающий дифференциальное уравнение сохраняется при действии $\mathfrak{a}$, то данное уравнение может быть записано через дифференциальные инварианты, отвечающие данному классу геометрических величин, и наконец,

3) Аффинная алгебра a является разрешимой алгеброй Ли размерности 2, а поэтому, в силу теоремы Ли-Бьянки ([4]), данное дифференциальное уравнение может быть проинтегрировано в квадратурах, если его порядок не превосходит 2 , а соответствующая геометрическая величина одномерна.

В качестве иллюстрации к указанной схеме, мы рассмотрим следующий пример.

Дифференциальные уравнения вида (см. [4], [1])

$$
y^{\prime \prime}=y^{\prime}+f(y)
$$

допускающие алгебру точечных симметрий a и тем самым интегрируемые в квадратурах, делятся на два класса:

(I) $\quad f=a(y+b)^{c}-\frac{2 c+2}{(c+3)^{2}}(y+b)$, где $a, b, c \in \mathbb{R}, \quad c \neq-3$, и

(II) $\quad f=a e^{b y}-\frac{2}{b}$, где $a, b \in \mathbb{R}, \quad b \neq 0$.

В начале мы рассмотрим класс $(I)$ и пусть $f=a y^{c}-\frac{2 x+2}{(c+3)^{2}} y$.

Тогда уравнение

$$
y^{\prime \prime}=y^{\prime}+a y^{c}-\frac{2 x+2}{(c+3)^{2}} y
$$

допускает алгебру точечных симметрий $\mathfrak{a}$ с образующими ([1]):

$$
A=\partial_{x}, \quad B=e^{k x} \partial_{x}+\frac{k+1}{2} e^{k x} u \partial_{u},
$$

где $k=\frac{1-c}{3+c}$.

Векторные поля $A$ и $B$ удовлетворяют коммутационному соотношению: $[A, B]=k B$.

Поэтому, положив

$$
X=e^{k x} \partial_{x}, \quad Y=-\frac{1}{k} \partial_{x},
$$

мы получим, что

$$
A=-k \bar{Y}, \quad B=\bar{X}
$$

и

$$
[X, Y]=X, \quad[\bar{X}, \bar{Y}]=\bar{X}
$$

Таким образом, алгебра симметрий является поднятием аффинного действия $\left\langle X=\partial_{x}, Y=x \partial_{x}\right\rangle$ на прямой. 
Аффинный параметр, который мы обозначим через $t$, находится из соотношения: $X(t)=1$. Из этого уравнения получаем, что $t=-\frac{1}{k} e^{-k x}$.

В качестве второй координаты $v$ на плоскости $\mathbb{R}^{2}$, мы выберем теперь первый интеграл векторного поля $\bar{X}=B$.

Соотношение $B(v)=0$ даёт дифференциальное уравнение $v_{x}+\alpha u v_{u}=0$, где $\alpha=\frac{k+1}{2}$. Поэтому, в качестве второй координаты, мы можем выбрать функцию $v=e^{-x} u^{\frac{1}{\alpha}}$. Мы имеем, $A(t)=-k t, A(v)=-v, B(t)=$ 1, $B(v)=0$.

Поэтому, в координатах $(t, v)$, алгебра симметрий $\langle\bar{X}, \bar{Y}\rangle$ порождена векторными полями

$$
\bar{X}=\partial_{t}, \quad \bar{Y}=t \partial_{t}+\frac{1}{k} v \partial_{v}
$$

Таким образом, решения дифференциального уравнения (1), является линейными аффинными геометрическими величинами. Несложно проверить, что они являются тензорами вида $h(t)\left(\partial_{x}\right)^{\otimes \frac{1}{k}}$, если $\frac{1}{k} \in \mathbb{Z}$.

Для дифференциальных уравнений класса $(I I)$ :

$$
y^{\prime \prime}=y^{\prime}+a e^{b y}-\frac{2}{b}
$$

алгебра точечных симметрий порождена векторными полями ([1]):

$$
A=\partial_{x}, \quad B=e^{-x} \partial_{x}+\frac{2}{b} e^{-x} u \partial_{u}
$$

удовлетворяющим коммутационному соотношению: $[A, B]=-B$.

Положим, $X=e^{-x} \partial_{x}, \quad Y=\partial_{x}$. Тогда, как и выше, $A=\bar{Y}, \quad B=\bar{X}$ и $[X, Y]=X$.

В качестве аффинного параметра, в данном случае, мы выберем функцию $t=e^{x}$, а в качестве первого интеграла векторного поля $B$ функцию $v=e^{-x} u^{\frac{b}{2}}$.

Тогда, $A(t)=t, \quad A(v)=-v, B(t)=1, \quad B(v)=0$. Поэтому,

$$
\bar{X}=\partial_{t}, \quad \bar{Y}=t \partial_{t}-v \partial_{v},
$$

в координатах $(t, v)$.

Таким образом, решения дифференциального уравнения (3) можно интерпретировать как дифференциальные формы на аффинной прямой. 


\section{2 Аффинные геометрические величины класса 1}

Одномерные аффинные геометрические величины являются сечениями расслоений ([2])

$$
\pi: \mathbb{R}^{2} \rightarrow \mathbb{R}
$$

где $\pi:(u, x,) \mapsto x$, в которые поднято действие аффинной алгебры Ли $\left\langle X=\partial_{x}, \quad Y=x \partial_{x}\right\rangle$.

Пусть

$$
\bar{X}=\partial_{x}+A(x, u) \partial_{u}, \quad \bar{Y}=x \partial_{x}+B(x, u) \partial_{u}
$$

такое поднятие действия этой алгебры в расслоение $\pi$.

В работе ([2]) показано, что эти поднятия имеют вид:

$$
\bar{X}=\partial_{x}-\frac{\varphi_{x}}{\varphi_{u}} \partial_{u}, \quad \bar{Y}=x \bar{X}
$$

где $\varphi(x, u)$ - произвольная гладкая функция, такая что, $\varphi_{u} \neq 0$.

В ([2]) также показано, что алгебра аффинных дифференциальных инвариантов для данного действия имеет следующую структуру. А именно,справедлива следующая теорема.

Теорема 1 Дифференииальные инварианты для аффинных геометрических величин класса 1 имеют два базисных инварианта:

-нулевого порядка $J_{0}=\varphi(x, u), u$

-второго порядка $J_{2}=\frac{\left(\frac{d^{2} \varphi}{d x^{2}}\right)}{\left(\frac{d \varphi}{d x}\right)^{2}}$, а все остальнье инварианты порождаются производными Трессе $J_{k+2}=\frac{D^{k} J_{2}}{D J_{0}^{k}}$.

\section{3 Дифференциальные уравнения и аффинные геометрические величины класса 1}

Мы рассматриваем одномерные аффинные геометрические величины и ассоциированные с ними обыкновенные дифференциальные уравнения.

В этом случае каждый аффинный дифференциальный инвариант может быть записан в виде:

$$
F\left(I, J, \frac{D J}{D I}, \ldots, \frac{D^{k} J}{D I^{k}}\right)
$$

где

$$
I=\varphi(x, u), \quad, J=\frac{d^{2} \varphi}{d x^{2}} /\left(\frac{d \varphi}{d x}\right)^{2},
$$


базисные инварианты, а $\varphi(x, u)$ - произвольная функция, удовлетворяющая условию $\varphi_{u} \neq 0$.

Дифференциальный инвариант (4) в свою очередь определяет обыкновенное дифференциальное уравнение

$$
F\left(I, J, \frac{D J}{D I}, \ldots, \frac{D^{k} J}{D I^{k}}\right)=0,
$$

порядка $k+2$, которое допускает алгебру Ли a симметрий с образующими:

$$
\bar{X}=\partial_{x}-\frac{\varphi_{x}}{\varphi_{u}} \partial_{u}, \quad \bar{Y}=x \bar{X}
$$

Аффинная алгебра $\mathfrak{a}$ - это разрешимая алгебра Ли размерности 2 , а поэтому, в силу теоремы Ли-Бьянки, всякое уравнение вида (5) при $k=0$ может быть явно проинтегрировано в квадратурах ([4], [1]).

Пусть теперь $k \geq 1$, и предположим, что уравнение (5), рассматриваемое как дифференциальное уравнение в производных Трессе, может быть проинтегрировано.

Тогда решения

$$
J=f(I)
$$

этого уравнения, рассматриваемые как обыкновенные дифференциальные уравнения второго порядка, интегрируются в квадратурах.

Рассмотрим, например, случай когда (5) является линейным дифференциальным уравнением 1-ого порядка относительно производной Трессе, то есть,

$$
\frac{D J}{D I}+A(I) J=B(I)
$$

Отметим, что в обычных производных уравнение (7) отвечает обыкновенному дифференциальному уравнению 3-го порядка.

Решая уравнение (7), мы находим 1-параметрическое семейство решений

$$
J=F(c, I)
$$

или $\frac{d^{2} \varphi}{d x^{2}}=F(c, \varphi)\left(\frac{d \varphi}{d x}\right)^{2}$, где с - некоторая постоянная.

Уравнения (8) являются уже дифференциальными 2-ого порядка, допускающими 2-мерную разрешимую алгебру симметрий, и следовательно интегрирующимися в квадратурах.

В качестве другого примера рассмотрим дифференциальные уравнения 4-ого порядка, отвечающие линейным дифференциальным уравнениям второго порядка с постоянными коэффициентами. Например:

$$
\frac{D^{2} J}{D I^{2}}+p \frac{D J}{D I}+q J=H(I)
$$


где p,q - некоторые постоянные.

Общее решение этого уравнения в случае различных характеристических корней, как хорошо известно, имеет вид

$$
J=K_{1}\left(I, C_{1}, C_{2}\right) e^{\lambda_{1} I}+K_{2}\left(I, C_{1}, C_{2}\right) e^{\lambda_{2} I},
$$

где функции $K_{1}$ и $K_{2}$ явно вычисляются через функцию $H$. Так например, для гармонического осциллятора,

$$
\begin{gathered}
\frac{D^{2} J}{D I^{2}}+\omega^{2} J=0, \\
J=C_{1} \cos (\omega I)+C_{2} \sin (\omega I),
\end{gathered}
$$

Обыкновенные дифференциальные уравнения (9) и (10), как и выше интегрируются в квадратурах.

Пример 1 Дифберенииальные уравнения вида:

$$
u_{4}-7 u_{1}^{-1} u_{2} u_{3}+8 u_{2}^{2}+W(u) u_{2}=A(u) u_{1}^{2}
$$

допускают аффинную алгебру симметрий $\mathfrak{a}$ с $\varphi=u$, для произвольньх функций $A$ и $W$. Будучи записанными в дифференииальных инвариантах, они приводят к линейным дифференииалъным уравнениям второго порядкa:

$$
\frac{D^{2} J}{D I^{2}}+W(I) J=A(I) .
$$

Если потенциал $W(I)$ интегрируем в смысле ([4]), то уравнение (11) может быть интегрировано в квадратурах, для произвольной функиии $A$.

Hапример, это так, когда $W(u)=C u^{-2}$ или $W(u)=C\left(u^{2}+p u+q\right)^{2}$.

Это также выполняется в случае, когда $W($ и) является решением стационарного уравнения Кортвега-де Вриза, или его высших аналогов ( см. [4]).

Пример 2 Дифференииальные уравнения вида:

$$
u_{1}^{2} u_{4}-7 u_{1} u_{2} u_{3}+8 u_{2}^{2}-u_{1}^{3} u_{3}+2 u_{1}^{2} u_{2}^{2}=a \frac{u_{2}^{k}}{u_{1}^{2 k-6}}-\frac{2(k+1)}{(k+3)^{2}} u_{1}^{4} u_{2}
$$

допускают афбинную алгебру симметрий $\mathfrak{a}$ с $\varphi=u$.

Записав (12) в дифференциальных инвариантах, мы приходим к дифференциальному уравнению:

$$
\frac{D^{2} J}{D I^{2}}=\frac{D J}{D I}+a J^{k}-\frac{2(k+1)}{(k+3)^{2}} J
$$


которое интегрируется в квадратурах ([1], [4]), и тем самым (12) такюе интегрируется в квадратурах. Примерами уравнений вида (12) являются следующие уравнения:

$$
\begin{array}{lrl}
k=a=2: & u_{1}^{2} u_{4}-7 u_{1} u_{2} u_{3}+8 u_{2}^{3}-u_{1}^{3} u_{3}+\frac{6}{25} u_{1}^{4} u_{2}=0, \\
k=3, a=8: & u_{1} u_{4}-7 u_{2} u_{3}-u_{1}^{2} u_{3}+2 u_{1} u_{2}^{2}+\frac{2}{9} u_{1}^{2} u_{2}=0 .
\end{array}
$$

\section{Список литературы}

1. S. V. Dushin, V. V. Lychagin. Symmetries of distributions and quadrature of ordinary differential equctions// Acta Appl. Math. 24: 1, (1991), P. 29-57.

2. Н. Г. Коновенко. Алгебри диференціальних інваріантів геометричних величин на афінній прямій// Вісник Київського національного університету. Серія:фіз.мат.науки, (2008), № 2, С. 9-16.

3. Н. Г. Коновенко. Дифференциальные инварианты и $\mathfrak{s l}_{2}$ - геометрии // Киів: "Наукова Думка"НАН України, (2013), 192 с.

4. A. Kushner, V. Lychagin, V. Roubtsov. Contact geometry and non-linear differential equations// Cambridge University Press, 2007, 516p.

Н. Г. Коновенко

http: / /orcid.org/0000-0002-8631-0688

ОНАПТ, Одесса, Украина

E-mail: konovenko@ukr.net

\section{Nadiia G. Konovenko}

\section{The quadratures and affine geometry on a line}

In this paper we apply affine geometry of the line in order to construct ordinary differential equations of the second order, integrable by quadratures. These equations are differential equations for affine geometric quantities admitting the affine group of symmetries. Due to Lie-Bianchi theorem such type equations are integrable by quadratures. We provide some examples to illustrate the method. 